\title{
On the anomaly of Balmer line profiles of A-type stars
}

\section{Fundamental binary systems ${ }^{\star}$}

\author{
B. Smalley ${ }^{1}$, R. B. Gardiner ${ }^{1 \star \star}$, F. Kupka ${ }^{2,3}$, and M. S. Bessell ${ }^{4}$ \\ 1 Astrophysics Group, School of Chemistry \& Physics, Keele University, Staffordshire, ST5 5BG, UK \\ e-mail: bs@astro.keele.ac.uk \\ 2 Institute for Astronomy, University of Vienna, Türkenschanzstr. 17, 1180 Vienna, Austria \\ e-mail: kupka@astro.univie.ac.at \\ 3 Astronomy Unit, School of Mathematical Sciences, Queen Mary, University of London, Mile End Road, \\ London, E1 4NS, UK e-mail: f.kupka@qmul .ac.uk \\ ${ }^{4}$ Research School of Astronomy and Astrophysics, Institute of Advanced Studies, The Australian National University, \\ Private Bag, Weston Creek PO, ACT 2611, Australia \\ e-mail: bessell@mso.anu.edu. au
}

Received 21 May 2002 / Accepted 10 September 2002

\begin{abstract}
In previous work, Gardiner et al. (1999) found evidence for a discrepancy between the $T_{\text {eff }}$ obtained from Balmer lines with that from photometry and fundamental values for A-type stars. An investigation into this anomaly is presented using Balmer line profiles of stars in binary system with fundamental values of both $T_{\text {eff }}$ and $\log g$. A revision of the fundamental parameters for binary systems given by Smalley \& Dworetsky (1995) is also presented. The $T_{\text {eff }}$ obtained by fitting $\mathrm{H} \alpha$ and $\mathrm{H} \beta$ line profiles is compared to the fundamental values and those obtained from uvby photometry. We find that the discrepancy found by Gardiner et al. (1999) for stars in the range $7000 \mathrm{~K} \lesssim T_{\text {eff }} \lesssim 9000 \mathrm{~K}$ is no longer evident.
\end{abstract}

Key words. stars: general - stars: atmospheres - convection - stars: binaries: eclipsing - stars: fundamental parameters

\section{Introduction}

Balmer lines are an important diagnostic of stellar atmospheric structure since they are formed at a wide range of depths within the atmosphere. In addition, the depth of formation of $\mathrm{H} \alpha$ is higher than that of $\mathrm{H} \beta$, thus observations of these profiles provide useful diagnostics (e.g. Gardiner 2000). Balmer profiles are relatively insensitive to surface gravity for stars cooler than $\sim 8000$ K (Gray 1992; see also Heiter et al. 2002). In addition, Balmer profiles are sensitive to the treatment of atmospheric convection (van't Veer-Menneret \& Megessier 1996; Castelli et al. 1997; Gardiner 2000; Heiter et al. 2002). For stars hotter than $\sim 8000 \mathrm{~K}$, the profiles are sensitive to both effective temperature and surface gravity. However, provided we know surface gravity from some other means (e.g. from eclipsing binary systems), we can use them to determine effective temperature.

In previous work, Smalley \& Kupka (1997, hereafter SK97) found no significant systematic problems with $u v b y$ and fundamental (and standard) stars. In fact, uvby was found to be very

Send offprint requests to: B. Smalley,

e-mail: bs@astro.keele.ac.uk

* Partly based on DENIS data obtained at the European Southern Observatory.

$\star \star$ Present Address: The Meteorological Office, Bracknell, UK. good for obtaining $T_{\text {eff }}$ and $\log g$. Using $\mathrm{H} \alpha$ and $\mathrm{H} \beta$ profiles, Gardiner et al. (1999, hereafter GKS99) found that both the Canuto \& Mazzitelli (1991, 1992) and standard Kurucz (1993) mixing-length theory without overshooting (MLTnoOV) (see Castelli et al. 1997) are both in agreement to within the uncertainties of the fundamental stars. Overshooting models were always clearly discrepant. However, GKS99 found some evidence for significant disagreement between all treatments of convection and fundamental values around $8000 \sim 9000 \mathrm{~K}$. In this region the effects of $\log g$ cannot be ignored. In GKS99, most of the $T_{\text {eff }}$ stars do not have fundamental values of $\log g$. Thus, possible $\log g$ bias could have occurred. In this paper, we use binary systems with fundamental values of $\log g$, determine revised fundamental values of $T_{\text {eff }}$ and compare the results with those from Balmer lines.

Binary systems with fundamental values of $\log g$, with in some cases fundamental values of $T_{\text {eff }}$, were discussed by Smalley \& Dworetsky (1995, hereafter SD95). Their list was limited to four systems, mainly due to lack of trigonometric parallax measurements. Fortunately, the Hipparcos mission (ESA, 1997) has provided trigonometric parallaxes for all of the binary systems considered by SD95. Thus we reevaluated 15 of those systems, using the methods of SD95, with 
slight modifications and a discussion of the sources of uncertainty.

Theoretical Balmer line profiles are compared to observations and the required values of $T_{\text {eff }}$ are derived which when used in a model atmosphere will predict the correct profiles. We have considered three models of convection: the standard mixing-length theory ATLAs9 models (Kurucz 1993; Castelli et al. 1997), with and without approximate convective overshooting, and modified atLas9 models based on the turbulent convection theory proposed by Canuto \& Mazzitelli (1991, 1992) and implemented by Kupka (1996).

\section{Effective temperatures of binary systems}

Eclipsing binary systems provide ideal test stars for comparing to models, since they enable us to obtain fundamental values of $T_{\text {eff }}$ and $\log g$. We can obtain fundamental values of $T_{\text {eff }}$ provided we know the apparent angular diameter $(\theta)$ and total integrated (bolometric) flux at the Earth $\left(f_{\oplus}\right)$. In the case of binary systems, where there are no direct measurements of angular diameters $(\theta)$, we can obtain them from the stellar radius $(R)$ and the parallax $(\pi)$ of the system (for details see SD95). Since the final $T_{\text {eff }}$ is twice as sensitive to $\theta$ than $f_{\oplus}$, it is critically important to obtain good values of $\theta$, which requires both accurate stellar radii measurements and accurate distance determinations.

Available spectrophotometry was taken from various sources (see Table 1), supplemented by 13-colour photometry fluxes from Johnson \& Mitchell (1975). All fluxes were placed on the Hayes \& Latham (1975) absolute flux scale. Unfortunately, only four stars had enough spectrophotometry. Many others, however, have at least $U B V$ or $U B V R I$ colours, which can be used to estimate the optical flux. Near-infrared fluxes were taken from the Gezari et al. (1987), 2MASS (Skrutskie et al. 1997) and DENIS (Epchtein et al. 1999) catalogues and placed on the Mountain et al. (1985) absolute flux scale.

Using a method similar to Petford et al. (1988), the total integrated flux in the optical region can be obtained. Theoretical $U B V$ or $U B V R I$ colours were compared to the observed values. The model which gave best agreement with the observations was then integrated from 3300-10000 $\AA$. While this method is not truly fundamental, the final integrated fluxes are not that sensitive to the choice of models. An uncertainly of $10 \%$ was adopted to accommodate errors in the photometry and model fluxes.

In a few cases, not even $U B V$ colours are available. However, these systems have Strömgren uvby photometry and hence $(B-V)$ can be obtained from $(b-y)$ using the relationship found by Crawford \& Barnes (1970). Petford et al. (1988) found that the $(B-V)$ index could be used to obtain the integrated flux from $3800-9000 \AA$, to within about $3 \%$ of that from reticon fluxes. Hence, in the absence of other observations, the Petford et al. empirical relationship is used, with an adopted uncertainty of $10 \%$, to be consistent with that adopted for fluxes obtained from $U B V$ and $U B V R I$ photometry.

Reddening has been determined using UVBYBETA (Moon 1985) and converted to $E(B-V)$ using the relationship
Table 1. Sources of flux measurements for binary systems.

\begin{tabular}{lcl}
\hline \hline \multicolumn{1}{c}{ Star } & HD & Flux sources \\
\hline 12 Per & 16739 & S2/68 B85 JM G87 \\
CD Tau & 34335 & S2/68 UBV 2MA \\
UX Men & 37513 & $(b-y)$ 2MA \\
$\beta$ Aur & 40183 & IUE J76 B85 K88 JM G87 \\
WW Aur & 46052 & S2/68 UBVRI 2MA \\
PV Pup & 62863 & $(b-y)$ MA \\
RS Cha & 75747 & $U B V$ \\
HS Hya & 90242 & $U B V$ MA DEN \\
RZ Cha & 93486 & $(b-y)$ DEN \\
$\gamma$ Vir & 110379 & IUE M78 B85 G92 K88 JM G87 \\
DM Vir & 123423 & $(b-y)$ \\
V624 Her & 161321 & IUE $U B V R I$ 2MA \\
V1143 Cyg & 185912 & S2/68 UBV \\
MY Cyg & 193637 & $U B V$ \\
$\delta$ Equ & 202275 & S2/68 B85 JM G87 \\
\hline
\end{tabular}

Key: IUE INES data from the IUE satellite, S2/68 Starlink STADAT data base (see Thompson et al. 1978, Carnochan 1979), J76 Jamar et al. (1976), M78 Macau-Hercot et al. (1978), B85 Burnashev (1985), G92 Glushneva et al. (1992), K88 Kharitonov et al. (1988), G87 Gezari et al. (1987), JM Johnson \& Mitchell (1975), 2MA Skrutskie et al. (1997), DEN Epchtein et al. (1999).

$E(B-V)=1.35 E(b-y)$ from Crawford (1975). Where necessary, the observed fluxes were de-reddened using the dereddening routine from DIPso (Howarth et al. 1997).

Following SD95, having obtained the combined $f_{\oplus}$ of the system, we can determine the $f_{\oplus}$ for each component by assuming that the difference in $f_{\oplus}$ between the two components is given by the observed magnitude difference, $\Delta m_{V}$. This is a reasonable approximation for a system with nearly identical components, but not necessarily valid for systems with markedly different spectral types. In these cases, we must apply the bolometric correction to obtain the difference in bolometric magnitudes in order to assign the appropriate $f_{\oplus}$ to each component. Bolometric corrections (BC) were calculated using the polynomials given by Flower (1996). Table 2 lists the values of fundamental radii and $\log g$ values and the observed $\Delta m_{V}$ and gives the results of $\theta, f_{\oplus}$ and $T_{\text {eff }}$ for each system. In most cases the values obtained using the Hipparcos distances are in agreement with that obtained from other means (e.g. Infrared Flux Method, uvby photometry, etc.) and other determinations (e.g. Jordi et al. 1997; Ribas et al. 1998). Three systems are anomalous. Two of them, PV Pup and DM Vir, have large uncertainties in their parallax measurements. The other system, HS Hya, appears to have a very good Hipparcos parallax. Torres et al. (1997) argue that an hitherto undetected third star in the system is likely to have affected the trigonometric parallax. Hence, these systems will not be used in our analysis.

Several of the systems considered here have measured infrared colours which enables us to employ the Infrared Flux Method (IRFM) developed by Blackwell \& Shallis (1977) to determine the mean $T_{\text {eff }}$ of the binary system, which can be compared to the "combined" $T_{\text {eff }}$ obtained using the fundamental method. The results from the IRFM are presented in Table 3. In most cases there is good agreement with the 
Table 2. Fundamental values of $T_{\text {eff }}$ for binary stars. The "combined" $T_{\text {eff }}$ values are given in italics.

\begin{tabular}{|c|c|c|c|c|c|c|c|c|c|}
\hline Star & $\begin{array}{l}\pi \\
\left(10^{-3 \prime \prime}\right)\end{array}$ & $\Delta m_{V}$ & & Radii & $\log g$ & $\begin{array}{l}\text { Sp. } \\
\text { Types }\end{array}$ & $\begin{array}{l}\theta \\
\left(10^{-3 \prime \prime}\right) \\
\end{array}$ & $\begin{array}{l}f_{\oplus} \\
\left(10^{-6} \mathrm{~W} \mathrm{~m}^{2}\right)\end{array}$ & $T_{\text {eff }}$ \\
\hline HD 16739 & $40.52 \pm 1.25$ & $0.57 \pm 0.04$ & \multicolumn{4}{|c|}{ BSF98 } & $0.788 \pm 0.034$ & $310 . \pm 20.0$ & $6220 \pm 168$ \\
\hline 12 Per & & & $\begin{array}{l}\text { A } \\
\text { B }\end{array}$ & $\begin{array}{l}1.58 \pm 0.05 \\
1.37 \pm 0.04\end{array}$ & $\begin{array}{l}4.16 \pm 0.03 \\
4.24 \pm 0.03\end{array}$ & $\begin{array}{l}\text { F8V } \\
\text { G2V }\end{array}$ & $\begin{array}{l}0.596 \pm 0.026 \\
0.517 \pm 0.022\end{array}$ & $\begin{array}{l}195 . \pm 12.8 \\
115 . \pm 5.06\end{array}$ & $\begin{array}{l}6371 \pm 176 \\
6000 \pm 143\end{array}$ \\
\hline HD 34335 & $13.66 \pm 1.64$ & $0.17 \pm 0.14$ & \multicolumn{4}{|c|}{ RJT99 } & $0.305 \pm 0.037$ & $45.0 \pm 5.00$ & $6177 \pm 410$ \\
\hline CD Tau & & & $\begin{array}{l}\text { A } \\
\text { B }\end{array}$ & $\begin{array}{l}1.798 \pm 0.017 \\
1.584 \pm 0.020\end{array}$ & $\begin{array}{l}4.087 \pm 0.010 \\
4.174 \pm 0.012\end{array}$ & $\begin{array}{l}\text { F6V } \\
\text { F6V }\end{array}$ & $\begin{array}{l}0.229 \pm 0.028 \\
0.201 \pm 0.024\end{array}$ & $\begin{array}{l}24.3 \pm 3.06 \\
20.7 \pm 1.61\end{array}$ & $\begin{array}{l}6110 \pm 415 \\
6260 \pm 397\end{array}$ \\
\hline $\begin{array}{l}\text { HD } 37513 \\
\text { UX Men }\end{array}$ & $9.93 \pm 0.62$ & $0.15 \pm 0.10$ & $\begin{array}{l}\text { A } \\
\text { B }\end{array}$ & $\begin{array}{r}\text { Al } \\
1.347 \pm 0.013 \\
1.274 \pm 0.013\end{array}$ & $\begin{array}{l}91 \\
4.272 \pm 0.009 \\
4.306 \pm 0.009\end{array}$ & $\begin{array}{l}\text { F8V } \\
\text { F8V }\end{array}$ & $\begin{array}{l}0.171 \pm 0.011 \\
0.124 \pm 0.008 \\
0.118 \pm 0.007\end{array}$ & $\begin{array}{l}14.0 \pm 2.00 \\
7.48 \pm 1.12 \\
6.52 \pm 0.54\end{array}$ & $\begin{array}{l}6151 \pm 293 \\
6171 \pm 302 \\
6130 \pm 233\end{array}$ \\
\hline $\begin{array}{l}\text { HD } 40183 \\
\beta \text { Aur }\end{array}$ & $39.72 \pm 0.78$ & $0.17 \pm 0.14$ & $\begin{array}{l}\text { A } \\
\text { B }\end{array}$ & $\begin{array}{l}\mathrm{N} \\
2.769 \pm 0.031 \\
2.627 \pm 0.029\end{array}$ & $\begin{array}{l}94 \\
3.930 \pm 0.010 \\
3.962 \pm 0.010\end{array}$ & $\begin{array}{l}\text { A1V } \\
\text { A1V }\end{array}$ & $\begin{array}{l}1.411 \pm 0.032 \\
1.023 \pm 0.023 \\
0.971 \pm 0.022\end{array}$ & $\begin{array}{l}4500 . \pm 379 \\
2430 . \pm 250 \\
2070 . \pm 139\end{array}$ & $\begin{array}{l}9077 \pm 217 \\
9131 \pm 257 \\
9015 \pm 182\end{array}$ \\
\hline $\begin{array}{l}\text { HD } 46052 \\
\text { WW Aur }\end{array}$ & $11.86 \pm 1.06$ & $0.19 \pm 0.23$ & $\begin{array}{l}\text { A } \\
\text { B }\end{array}$ & $\begin{array}{r}\text { AI } \\
1.883 \pm 0.038 \\
1.883 \pm 0.038\end{array}$ & $\begin{array}{l}91 \\
4.187 \pm 0.019 \\
4.143 \pm 0.018\end{array}$ & $\begin{array}{l}\mathrm{A} 5 \mathrm{~m} \\
\mathrm{~A} 7 \mathrm{~m}\end{array}$ & $\begin{array}{l}0.294 \pm 0.027 \\
0.208 \pm 0.019 \\
0.208 \pm 0.019\end{array}$ & $\begin{array}{l}108 . \pm 12.0 \\
58.7 \pm 8.65 \\
49.3 \pm 5.01\end{array}$ & $\begin{array}{l}7827 \pm 419 \\
7993 \pm 470 \\
7651 \pm 401\end{array}$ \\
\hline $\begin{array}{l}\text { HD } 62863 \\
\text { PV Pup }\end{array}$ & $8.10 \pm 5.88$ & $0.05 \pm 0.27$ & $\begin{array}{l}\text { A } \\
\text { B }\end{array}$ & $\begin{array}{r}\mathrm{Al} \\
1.542 \pm 0.018 \\
1.499 \pm 0.018\end{array}$ & $\begin{array}{l}91 \\
4.257 \pm 0.010 \\
4.278 \pm 0.011\end{array}$ & $\begin{array}{l}\mathrm{A} 8 \mathrm{~V} \\
\mathrm{~A} 8 \mathrm{~V}\end{array}$ & $\begin{array}{l}0.162 \pm 0.118 \\
0.116 \pm 0.084 \\
0.113 \pm 0.082\end{array}$ & $\begin{array}{l}43.0 \pm 6.00 \\
22.0 \pm 4.07 \\
21.0 \pm 2.41\end{array}$ & $\begin{array}{l}8372 \pm 3053 \\
8361 \pm 3060 \\
8383 \pm 3053\end{array}$ \\
\hline $\begin{array}{l}\text { HD } 75747 \\
\text { RS Cha }\end{array}$ & $10.23 \pm 0.46$ & $0.01 \pm 0.16$ & $\begin{array}{l}\text { A } \\
\text { B }\end{array}$ & $\begin{array}{r}\text { AI } \\
2.137 \pm 0.055 \\
2.338 \pm 0.055\end{array}$ & $\begin{array}{l}991 \\
4.047 \pm 0.023 \\
3.961 \pm 0.021\end{array}$ & $\begin{array}{l}\mathrm{A} 8 \mathrm{~V} \\
\mathrm{~A} 8 \mathrm{~V}\end{array}$ & $\begin{array}{l}0.302 \pm 0.015 \\
0.203 \pm 0.011 \\
0.223 \pm 0.011\end{array}$ & $\begin{array}{l}88.0 \pm 9.00 \\
44.2 \pm 5.56 \\
43.8 \pm 3.21\end{array}$ & $\begin{array}{l}7342 \pm 266 \\
7525 \pm 307 \\
7178 \pm 225\end{array}$ \\
\hline $\begin{array}{l}\text { HD } 90242 \\
\text { HS Hya }\end{array}$ & $11.04 \pm 0.88$ & $0.17 \pm 0.09$ & $\begin{array}{l}\text { A } \\
\text { B }\end{array}$ & $\begin{array}{r}\mathrm{TC} \\
1.275 \pm 0.007 \\
1.216 \pm 0.007\end{array}$ & $\begin{array}{l}97 \\
4.326 \pm 0.006 \\
4.354 \pm 0.006\end{array}$ & $\begin{array}{l}\text { F5V } \\
\text { F5V }\end{array}$ & $\begin{array}{l}0.181 \pm 0.014 \\
0.131 \pm 0.010 \\
0.125 \pm 0.010\end{array}$ & $\begin{array}{l}14.0 \pm 1.40 \\
7.55 \pm 0.80 \\
6.45 \pm 0.40\end{array}$ & $\begin{array}{l}5985 \pm 282 \\
6028 \pm 290 \\
5936 \pm 255\end{array}$ \\
\hline $\begin{array}{l}\text { HD } 93486 \\
\text { RZ Cha }\end{array}$ & $5.43 \pm 0.63$ & $0.00 \pm 0.14$ & $\begin{array}{l}\text { A } \\
\text { B }\end{array}$ & $\begin{array}{r}\text { AI } \\
2.264 \pm 0.017 \\
2.264 \pm 0.017\end{array}$ & $\begin{array}{l}91 \\
3.909 \pm 0.009 \\
3.907 \pm 0.010\end{array}$ & $\begin{array}{l}\text { F5V } \\
\text { F5V }\end{array}$ & $\begin{array}{l}0.162 \pm 0.019 \\
0.114 \pm 0.013 \\
0.114 \pm 0.013\end{array}$ & $\begin{array}{l}15.0 \pm 2.00 \\
7.50 \pm 1.11 \\
7.50 \pm 0.60\end{array}$ & $\begin{array}{l}6440 \pm 432 \\
6440 \pm 444 \\
6440 \pm 396\end{array}$ \\
\hline $\begin{array}{l}\text { HD } 110379 \\
\gamma \text { Vir }\end{array}$ & $84.53 \pm 1.18$ & $0.00 \pm 0.04$ & $\begin{array}{l}\text { A } \\
\text { B }\end{array}$ & $\begin{array}{l}\text { PC } \\
1.35 \pm 0.16 \\
1.35 \pm 0.16\end{array}$ & $\begin{array}{l}80 \\
4.21 \pm 0.017 \\
4.21 \pm 0.017\end{array}$ & $\begin{array}{l}\text { F0V } \\
\text { F0V }\end{array}$ & $\begin{array}{l}1.502 \pm 0.179 \\
1.062 \pm 0.127 \\
1.062 \pm 0.127\end{array}$ & $\begin{array}{l}1960 . \pm 157 \\
978 . \pm 80.5 \\
978 . \pm 41.3\end{array}$ & $\begin{array}{l}7143 \pm 450 \\
7143 \pm 451 \\
7143 \pm 433\end{array}$ \\
\hline $\begin{array}{l}\text { HD } 123423 \\
\text { DM Vir }\end{array}$ & $2.91 \pm 1.25$ & $0.00 \pm 0.14$ & $\begin{array}{l}\text { A } \\
\text { B }\end{array}$ & $\begin{array}{r}\mathrm{L} \\
1.763 \pm 0.017 \\
1.763 \pm 0.017\end{array}$ & $\begin{array}{l}96 \\
4.108 \pm 0.009 \\
4.106 \pm 0.009\end{array}$ & $\begin{array}{l}\text { F7V } \\
\text { F7V }\end{array}$ & $\begin{array}{l}0.068 \pm 0.029 \\
0.048 \pm 0.021 \\
0.048 \pm 0.021\end{array}$ & $\begin{array}{l}6.00 \pm 1.00 \\
3.00 \pm 0.53 \\
3.00 \pm 0.28\end{array}$ & $\begin{array}{l}7928 \pm 1735 \\
7928 \pm 1740 \\
7928 \pm 1714\end{array}$ \\
\hline $\begin{array}{l}\text { HD } 161321 \\
\text { V624 Her }\end{array}$ & $6.93 \pm 0.74$ & $0.79 \pm 0.12$ & $\begin{array}{l}\text { A } \\
\text { B }\end{array}$ & $\begin{array}{r}\text { AI } \\
3.030 \pm 0.034 \\
2.209 \pm 0.034\end{array}$ & $\begin{array}{l}91 \\
3.834 \pm 0.010 \\
4.024 \pm 0.014\end{array}$ & $\begin{array}{l}\text { A3m } \\
\text { A7V }\end{array}$ & $\begin{array}{l}0.242 \pm 0.026 \\
0.195 \pm 0.021 \\
0.142 \pm 0.015\end{array}$ & $\begin{array}{l}89.0 \pm 9.00 \\
60.0 \pm 6.44 \\
29.0 \pm 2.55\end{array}$ & $\begin{array}{l}8222 \pm 489 \\
8288 \pm 497 \\
8092 \pm 474\end{array}$ \\
\hline $\begin{array}{l}\text { HD } 185912 \\
\text { V1143 Cyg }\end{array}$ & $25.12 \pm 0.56$ & $0.07 \pm 0.11$ & $\begin{array}{l}\text { A } \\
\text { B }\end{array}$ & $\begin{array}{r}\text { AI } \\
1.346 \pm 0.023 \\
1.323 \pm 0.023\end{array}$ & $\begin{array}{l}91 \\
4.323 \pm 0.016 \\
4.324 \pm 0.016\end{array}$ & $\begin{array}{l}\text { F5V } \\
\text { F5V }\end{array}$ & $\begin{array}{l}0.441 \pm 0.012 \\
0.315 \pm 0.009 \\
0.309 \pm 0.009\end{array}$ & $\begin{array}{l}110 . \pm 11.0 \\
56.8 \pm 6.32 \\
53.2 \pm 3.38\end{array}$ & $\begin{array}{l}6418 \pm 184 \\
6441 \pm 201 \\
6393 \pm 136\end{array}$ \\
\hline $\begin{array}{l}\text { HD } 193637 \\
\text { MY Cyg }\end{array}$ & $3.79 \pm 0.87$ & $0.03 \pm 0.16$ & $\begin{array}{l}\text { A } \\
\text { B }\end{array}$ & $\begin{array}{r}\text { AI } \\
2.193 \pm 0.050 \\
2.193 \pm 0.050\end{array}$ & $\begin{array}{l}91 \\
4.008 \pm 0.021 \\
4.014 \pm 0.021\end{array}$ & $\begin{array}{l}\text { F0m } \\
\text { F0m }\end{array}$ & $\begin{array}{l}0.109 \pm 0.025 \\
0.077 \pm 0.018 \\
0.077 \pm 0.018\end{array}$ & $\begin{array}{l}12.2 \pm 1.22 \\
6.17 \pm 0.76 \\
6.00 \pm 0.43\end{array}$ & $\begin{array}{l}7434 \pm 877 \\
7459 \pm 891 \\
7408 \pm 865\end{array}$ \\
\hline $\begin{array}{l}\text { HD } 202275 \\
\delta \text { Equ }\end{array}$ & $54.11 \pm 0.85$ & $0.00 \pm 0.07$ & $\begin{array}{l}\text { A } \\
\text { B }\end{array}$ & $\begin{array}{l}1.22 \pm 0.03 \\
1.22 \pm 0.03\end{array}$ & $\begin{array}{l}80 \\
4.34 \pm 0.02 \\
4.34 \pm 0.02\end{array}$ & $\begin{array}{l}\text { F7V } \\
\text { F7V }\end{array}$ & $\begin{array}{l}0.869 \pm 0.025 \\
0.614 \pm 0.018 \\
0.614 \pm 0.018\end{array}$ & $\begin{array}{l}420 . \pm 30.0 \\
210 . \pm 16.5 \\
210 . \pm 8.90\end{array}$ & $\begin{array}{l}6393 \pm 147 \\
6393 \pm 156 \\
6393 \pm 115\end{array}$ \\
\hline
\end{tabular}

Key: BSF98 Barlow et al. (1998); RJT99 Ribas et al. (1999); NJ94 Nordström \& Johansen (1994b); AND91 Andersen (1991); TOR97 Torres et al. (1997); POP80 Popper (1980); LAT96 Latham et al. (1996).

fundamental values. However, for three systems there is significant disagreement:

PV Pup: as noted above the fundamental value is unreliable, but the IRFM value is also significantly hotter than that obtained from $u v b y \beta$ photometry $(7140 \mathrm{~K})$. Thus, we conclude that the IRFM result is unreliable, possibly due to the uncertainty of the optical flux, which was obtained from $b-y$ alone.

$\gamma$ Vir: the IRFM result is cooler than the fundamental value, but both agree to within the relatively large errorbar of the fundamental value. Interestingly, SD95 obtained a value of $T_{\text {eff }}=6750 \pm 470 \mathrm{~K}$ using the van Altena et al. (1991) trigonometric parallax, which is in excellent agreement with the IRFM.

V624 Her: the IRFM is cooler than the fundamental value. The fundamental value is, however, in agreement with Popper (1984) and Ribas et al. (1998).

Having obtained fundamental $T_{\text {eff }}$ values for the binary systems, we now present observations of the Balmer lines of these systems and the results of fitting profiles using models with differing treatments of convection. We will also refer to the IRFM values and values of $T_{\text {eff }}$ obtained from $u v b y$ photometry. 
Table 3. Effective temperatures obtained from the Infrared Flux Method (IRFM) for the binary systems. The values are the "combined" flux $T_{\text {eff }}$.

\begin{tabular}{lll}
\hline \hline Star & HD & $T_{\text {eff }}$ \\
\hline 12 Per & 16739 & $6275 \pm 229$ \\
CD Tau & 34335 & $5985 \pm 203$ \\
UX Men & 37513 & $6204 \pm 244$ \\
$\beta$ Aur & 40183 & $8858 \pm 221$ \\
WW Aur & 46052 & $7795 \pm 227$ \\
PV Pup & 62863 & $7759 \pm 313$ \\
HS Hya & 90242 & $6256 \pm 181$ \\
RZ Cha & 93486 & $6404 \pm 260$ \\
$\gamma$ Vir & 110379 & $6747 \pm 189$ \\
V624 Her & 161321 & $7710 \pm 218$ \\
$\delta$ Equ & 202275 & $6293 \pm 162$ \\
\hline
\end{tabular}

\section{Observations}

The $\mathrm{H} \alpha$ and $\mathrm{H} \beta$ observations were made at the Observatorio del Roque de los Muchachos, La Palma using the RichardsonBrealey Spectrograph on the $1.0 \mathrm{~m}$ Jacobus Kapteyn Telescope (JKT) in 1997 October/November. A $24001 \mathrm{~mm}^{-1}$ holographic grating was used and a $1124 \times 1124$ pixel Tek CCD, giving a resolution of $0.4 \AA$ FwHM. Further $\mathrm{H} \alpha$ and $\mathrm{H} \beta$ observations were made at the Mount Stromlo Observatory, Australia in February 2000 using the Cassegrain Spectrograph on the ANU 74 inch telescope. A $12001 \mathrm{~mm}^{-1}$ blazed grating was used, giving a resolution of $0.35 \AA$ FWHM. Additional $\mathrm{H} \beta$ profiles were taken from the work of SD95.

The data reduction of the profiles taken in 1997 and 2000 was performed using the Starlink всномор software package (Mills et al. 1997). In most cases the final spectra had a signalto-noise ratio in excess of 100:1. Instrumental sensitivity variations were removed from the $\mathrm{H} \alpha$ profiles by comparing to observations of stars with intrinsically narrow Balmer profiles, for example early-B or O type stars and $\mathrm{G}$ type stars, and the $\mathrm{H} \beta$ profiles were normalized such that the observed profile of Vega agreed to a model with $T_{\text {eff }}=9550 \mathrm{~K}, \log g=3.95$, $[\mathrm{M} / \mathrm{H}]=-0.5$ (Castelli \& Kurucz 1994) and the standard profiles of Peterson (1969).

\section{Effective temperatures from Balmer line profiles}

The observed Balmer line profiles are fitted here to model spectra to compare the derived $T_{\text {eff }}$ with that from fundamental methods. One of our main aims is to investigate the performance of different models of convection. The following convection models were used, using solar-metallicity Kurucz ATLAS models:

1. Standard ATLAs9 (Kurucz 1993) models using mixing length theory (MLT) without convective overshooting. The value of the MLT parameter $\alpha$ is the standard value of 1.25 . These will be referred to as MLT_noOV 1.25 models in this paper.

2. Standard ATLAS 9 models using MLT without convective overshooting. The value of the MLT parameter $\alpha$ is 0.5 . These will be referred to as MLT_noOV 0.5 models.
3. Standard ATLAS9 models using MLT with approximate overshooting. The value of the MLT parameter $\alpha$ used is 1.25 . These will be referred to as MLT_OV 1.25 models.

4. Standard ATLAS 9 models using MLT with approximate convective overshooting. The value of the MLT parameter $\alpha$ used is 0.5 . These will be referred to as MLT_OV 0.5 models.

5. Modified atlas 9 models using the Canuto \& Mazzitelli (1991, 1992) model of turbulent convection. These will be referred to as the CM models.

The synthetic spectra were calculated using UCLSYN (Smith 1992; Smalley et al. 2001) which includes Balmer line profiles calculated using the Stark-broadening tables of Vidal et al. (1973) and metal absorption lines from the Kurucz \& Bell (1995) linelist. This routine is based on the BALMER routine (Peterson 1969; Kurucz 1993). The spectra were rotationally broadened as necessary and instrumental broadening was applied with $F W H M=0.4 \AA$ to match the resolution of the observations. The synthetic spectra were normalized at $\pm 100 \AA$ to match the observations. The values of $T_{\text {eff }}$ were obtained by fitting model profiles to the observations using the leastsquare differences. A microturbulence of $2 \mathrm{~km} \mathrm{~s}^{-1}$ was assumed throughout for both the model atmosphere line opacities and spectrum synthesis.

Two stars, HD 46052 and HD 161 321, are Am stars, with $[\mathrm{M} / \mathrm{H}]=0.23$ and 0.29 , respectively, calculated from the $\delta m_{0}$ calibrations, derived by Berthet (1990) and Smalley (1992). For these stars Balmer profile fits using the solar abundance grid model atmospheres gave results for $T_{\text {eff }}$ which were around $50 \sim 100 \mathrm{~K}$ hotter, compared to models with the appropriate $[\mathrm{M} / \mathrm{H}]$.

The Balmer line profiles become increasingly less sensitive to $\log g$ below $\sim 8000 \mathrm{~K}$ thus any errors in the values of $\log g$ used will have no significant effect on the results. Hotter than $\sim 8000 \mathrm{~K}$ the Balmer profiles become progressively more sensitive to $\log g$, making it important to have a fundamental value of $\log g$ with a small error. For example, at $8000 \mathrm{~K}$, a change in assumed $\log g$ of 0.5 dex would have the effect to changing the temperature of the model profile an observation is fit to by $\sim 150 \mathrm{~K}$.

The best fitting values of $T_{\text {eff }}$ for the binary stars are given in Table 4 , for the 5 convection models listed above. Figures 1 and 2 show the variation of $\Delta T_{\text {eff }}=T_{\text {eff }}$ (Balmer) $-T_{\text {eff }}$ (fund) against $T_{\text {eff }}$ (fund) for $\mathrm{H} \alpha$ and $\mathrm{H} \beta$, respectively. To within the uncertainties, the $\mathrm{CM}$ results show no significant variation with $T_{\text {eff }}$ (fund) for either $\mathrm{H} \alpha$ or $\mathrm{H} \beta$. The discrepancy around $8000 \mathrm{~K}$ noted by GKS99 is not evident. Even the two anomalous $\mathrm{H} \alpha$ points just hotter than $8000 \mathrm{~K}$ for V624 Her can be brought into agreement if the IRFM $T_{\text {eff }}$ is used (see Sect. 2). The MLT_noOV results are in broad agreement with those for CM, but with the $\alpha=0.5$ models giving better agreement around $8000 \mathrm{~K}$ relative to $\alpha=1.25$ and CM models. Contrary to GKS99, who reported that F-type stars might require models with $\alpha \geq 1.25$ (see their Fig. 9), we find that the binary systems do not support this. Overall, $\alpha=0.5$ models are preferred to those with higher values. The MLT_OV models are generally more discrepant, yielding too high values of $T_{\text {eff }}$ (and even 

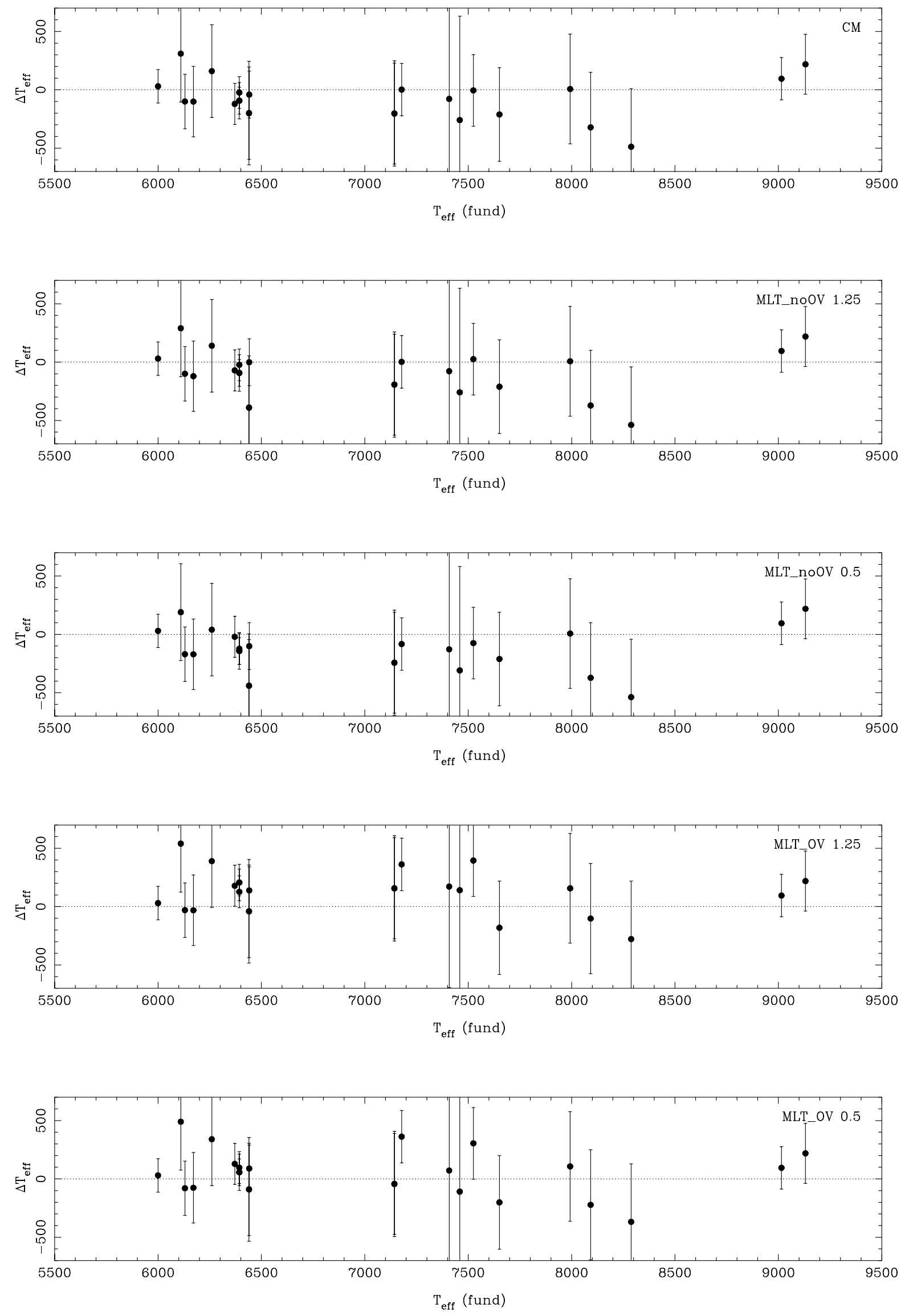

Fig. 1. Comparison between $T_{\text {eff }}$ calculated from Balmer line profiles $\mathrm{H} \alpha$ to those derived from fundamental methods for the five convection models. $\Delta T_{\text {eff }}=T_{\text {eff }}$ (Balmer) $-T_{\text {eff }}$ (fund) is plotted against $T_{\text {eff }}$ (fund). 

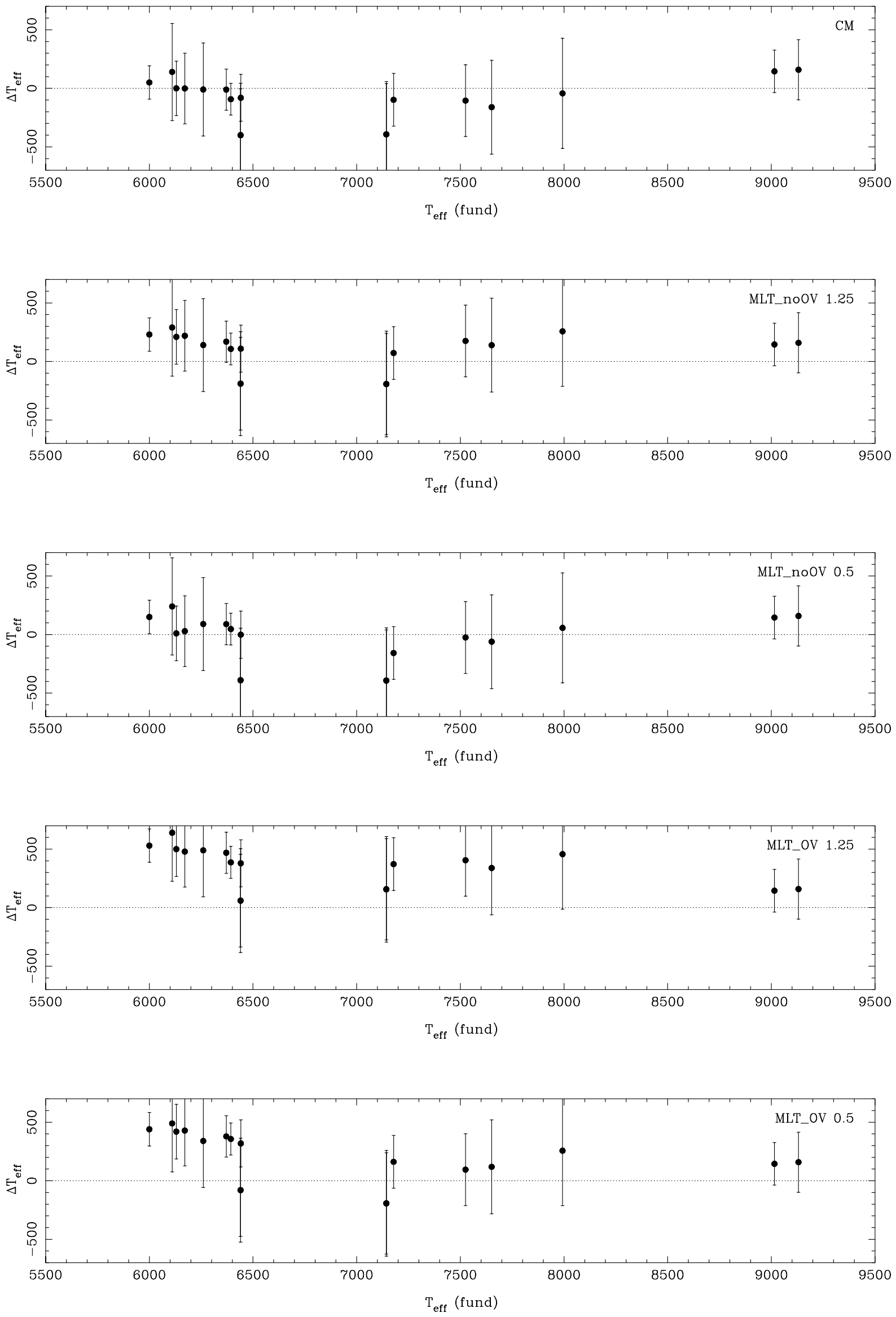

Fig. 2. Same as Fig. 1, but for $\mathrm{H} \beta$. 
Table 4. $T_{\text {eff }}$ obtained from Balmer line profiles for binary systems with known values of $\log g$.

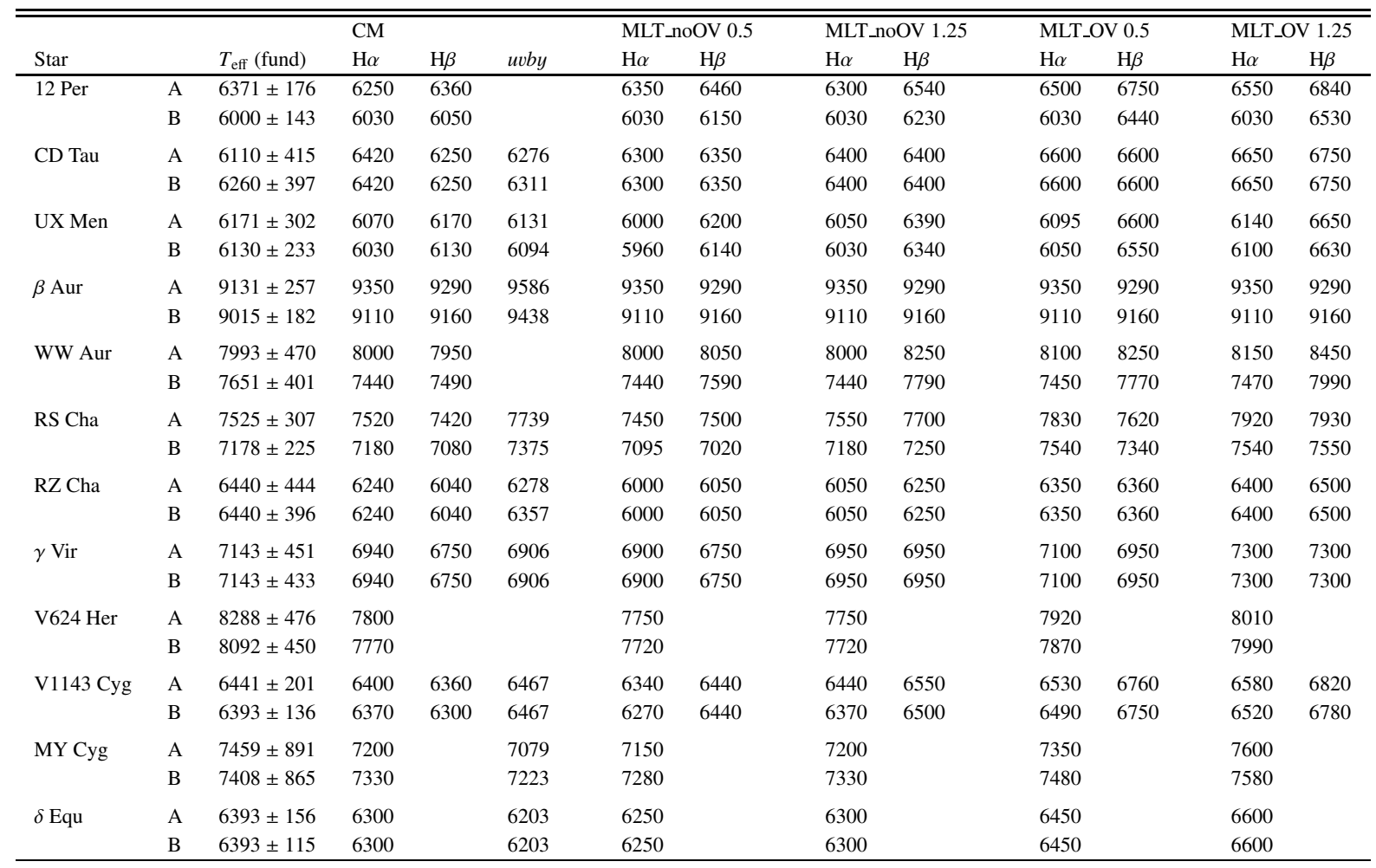

larger ones for $\mathrm{H} \beta$, if $\alpha=1.25$ is used rather than 0.5 ), as found previously by GKS99. Note also the systematic difference between $\mathrm{H} \alpha$ and $\mathrm{H} \beta$ for $\alpha=1.25$ MLT_noOV models, which is even more pronounced for the MLT_OV models.

\subsection{The apparent A-star anomaly}

The use of stars with fundamental values of both $T_{\text {eff }}$ and $\log g$ has failed to support the apparent anomaly around $8000 \mathrm{~K}$ found by GKS99, in which Balmer profiles gave progressively lower values of $T_{\text {eff }}$ compared to fundamental values. However, there are too few stars within the $T_{\text {eff }}$ range $8000-9000 \mathrm{~K}$ to fully explore this region. In addition, GKS99 found that four fundamental $T_{\text {eff }}$ stars showed the anomaly: HR 4534, HR 6556, HR 7557, and HR 8728. In order to be sure that there is no anomaly in the Balmer line profiles, we need to explain why these stars might appear anomalous. We have also included HR 2421 which is just hotter than $9000 \mathrm{~K}$, but find that this is in agreement with its fundamental and IRFM $T_{\text {eff }}$.

Table 5 summarizes the values of $T_{\text {eff }}$ obtained from CM models and uvby photometry, the IRFM and by fitting to $\mathrm{H} \alpha$ and $\mathrm{H} \beta$ profiles. We have allowed both $T_{\text {eff }}$ and $\log g$ to vary in order to obtain the best least-squares fit (see Figs. 3 and 4). Values of $\log g$ are also given as obtained from $u v b y$ photometry, as well as those adopted by GKS99.

The rapidly rotating star HR 7557 has recently been studied by van Belle et al. (2001) using interferometry. Their analysis revealed the oblateness of the star and gave revised angular diameter values and a new determination of fundamental $T_{\text {eff }}=7680 \pm 90 \mathrm{~K}$. This is significantly cooler than the previous determination, but in accord with that inferred from the IRFM. As such, the $T_{\text {eff }}$ from $\mathrm{H} \alpha$ and $\mathrm{H} \beta$ are no longer significantly discrepant. In fact the new $T_{\text {eff }}$ is in agreement with that given in Gray (1992) for a A7V star. It is certainly possible that revision to the other fundamental stars could occur once new interferometric measurements are obtained, especially HR 6556 which has a similar $v \sin i$ and might be expected to exhibit significant oblateness. Thus, the anomalies for HR 6556 and HR 7557 can be explained in terms of their rapid rotation. However, the earlier angular diameter measurements are not necessarily the source of any discrepancy, since the new Vega (HR 7001) value obtained by Ciardi et al. (2001) is in excellent agreement with that of Hanbury Brown et al. (1974).

The two other stars, HR 4534 and HR 8728, have lower $v \sin i$ values, but are the two most discrepant stars in the GKS99 sample. Unless the fundamental values are truly wrong there must be some other reason for the discrepancy. The IRFM values both point to a slightly cooler $T_{\text {eff }}$, but even then the discrepancy is $\sim 300 \mathrm{~K}$. However, in this temperature region the Balmer lines are near their maximum strength and sensitive to $\log g$. It is certainly possible that a small error in adopted $\log g$ could lead to a large error in $T_{\text {eff }}$ obtained from Balmer profiles. In fact, using the values of $\log g$ obtained from $u v b y$ photometry requires $T_{\text {eff }} \simeq 7900 \mathrm{~K}$ in order to fit the observed $\mathrm{H} \alpha$ profiles for both stars and $T_{\text {eff }} \simeq 8100 \mathrm{~K}$ to fit the $\mathrm{H} \beta$ profile of HR 4534. In addition, the Balmer profiles change little with relatively large changes in $T_{\text {eff. }}$ Thus, we conclude that the two stars are not discrepant, due to the low sensitivity of Balmer lines with respect to changes in $T_{\text {eff }}$ and both sensitivity to, and the uncertainty in, the surface gravity for these stars.

In general, for stars hotter than $8000 \mathrm{~K}$ the sensitivity to $\log g$ prevents us from using Balmer line profiles to obtain 
Table 5. Early A-stars with fundamental values of $T_{\text {eff }}$, but not $\log g$.

\begin{tabular}{|c|c|c|c|c|c|c|c|c|c|c|c|}
\hline \multirow[t]{2}{*}{ HR } & \multirow[t]{2}{*}{ star } & \multirow{2}{*}{$\begin{array}{l}v \sin i \\
\left(\mathrm{~km} \mathrm{~s}^{-2}\right)\end{array}$} & \multirow{2}{*}{$\begin{array}{l}T_{\text {eff }} \\
\text { Fund }\end{array}$} & \multirow{2}{*}{$\begin{array}{c}\log g \\
\text { GKS99 }\end{array}$} & \multicolumn{2}{|c|}{ CM $u v b y$} & \multirow{2}{*}{$\begin{array}{c}T_{\text {eff }} \\
\text { IRFM }\end{array}$} & \multicolumn{2}{|c|}{$\mathrm{H} \alpha$} & \multicolumn{2}{|c|}{$\overline{\mathrm{H} \beta}$} \\
\hline & & & & & $T_{\text {eff }}$ & $\log g$ & & $T_{\text {eff }}$ & $\log g$ & $T_{\text {eff }}$ & $\log g$ \\
\hline 2421 & $\gamma \mathrm{Gem}$ & 45 & $9220 \pm 330$ & 3.5 & $9250 \pm 460$ & $3.56 \pm 0.30$ & $9040 \pm 86$ & $9220 \pm 300$ & $3.40 \pm 0.2$ & $9060 \pm 250$ & $3.52 \pm 0.2$ \\
\hline 4534 & $\beta$ Leo & 122 & $8870 \pm 350$ & 4.1 & $8770 \pm 300$ & $4.32 \pm 0.11$ & $8660 \pm 60$ & $8370 \pm 400$ & $3.77 \pm 0.2$ & $8450 \pm 350$ & $4.07 \pm 0.2$ \\
\hline 6556 & $\alpha \mathrm{Oph}$ & 240 & $7960 \pm 330$ & 3.8 & $7940 \pm 210$ & $3.80 \pm 0.17$ & $7883 \pm 63$ & $7510 \pm 100$ & $3.69 \pm 0.3$ & $7580 \pm 150$ & $3.42 \pm 0.6$ \\
\hline 7557 & $\alpha \mathrm{Aql}$ & 245 & $7990 \pm 210$ & 4.2 & $7840 \pm 200$ & $4.18 \pm 0.17$ & $7588 \pm 73$ & $7420 \pm 100$ & $4.17 \pm 0.3$ & $7450 \pm 150$ & $4.38 \pm 0.6$ \\
\hline 8728 & $\alpha$ PsA & 85 & $8760 \pm 310$ & 4.2 & $8890 \pm 320$ & $4.30 \pm 0.10$ & $8622 \pm 86$ & $8340 \pm 400$ & $3.87 \pm 0.2$ & & \\
\hline
\end{tabular}

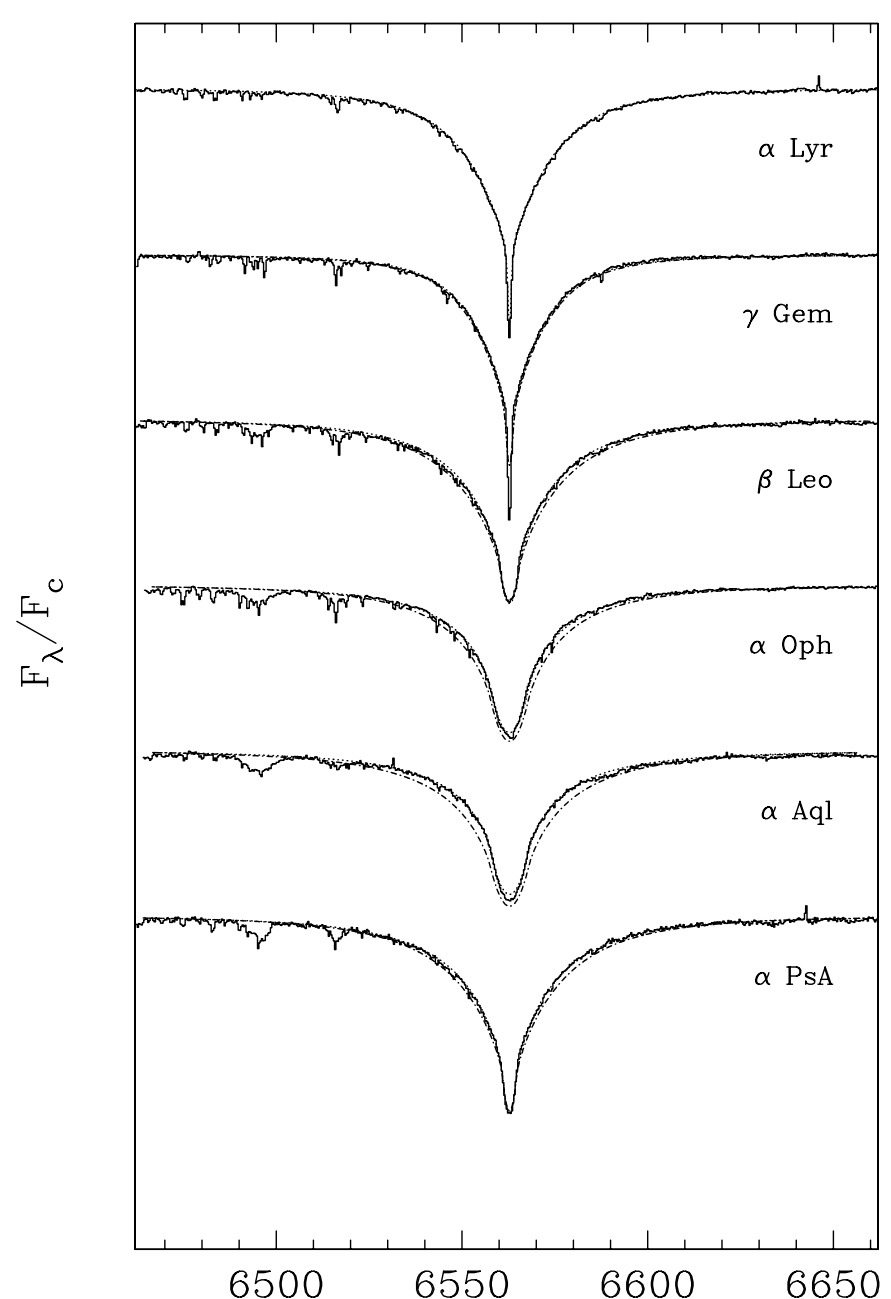

$\lambda(\AA)$

Fig. 3. H $\alpha$ profiles of A stars. The continuous line is the observed profile, while the dotted line is the synthetic profile for best fitting parameters given in Table 5. The dash-dot line is that for profiles calculated for the fundamental parameters. The profiles for Vega are given for reference.

values of $T_{\text {eff }}$ to the accuracy required for the present task, unless we have accurate fundamental values of $\log g$. However, until we do have stars with accurate fundamental $\log g$ values, we cannot be totally sure that there is not a problem with the model predictions in this $T_{\text {eff }}$ region.

\section{Conclusion}

The availability of the Hipparcos parallax measurements has enabled the list of stars with fundamental values of both $T_{\text {eff }}$

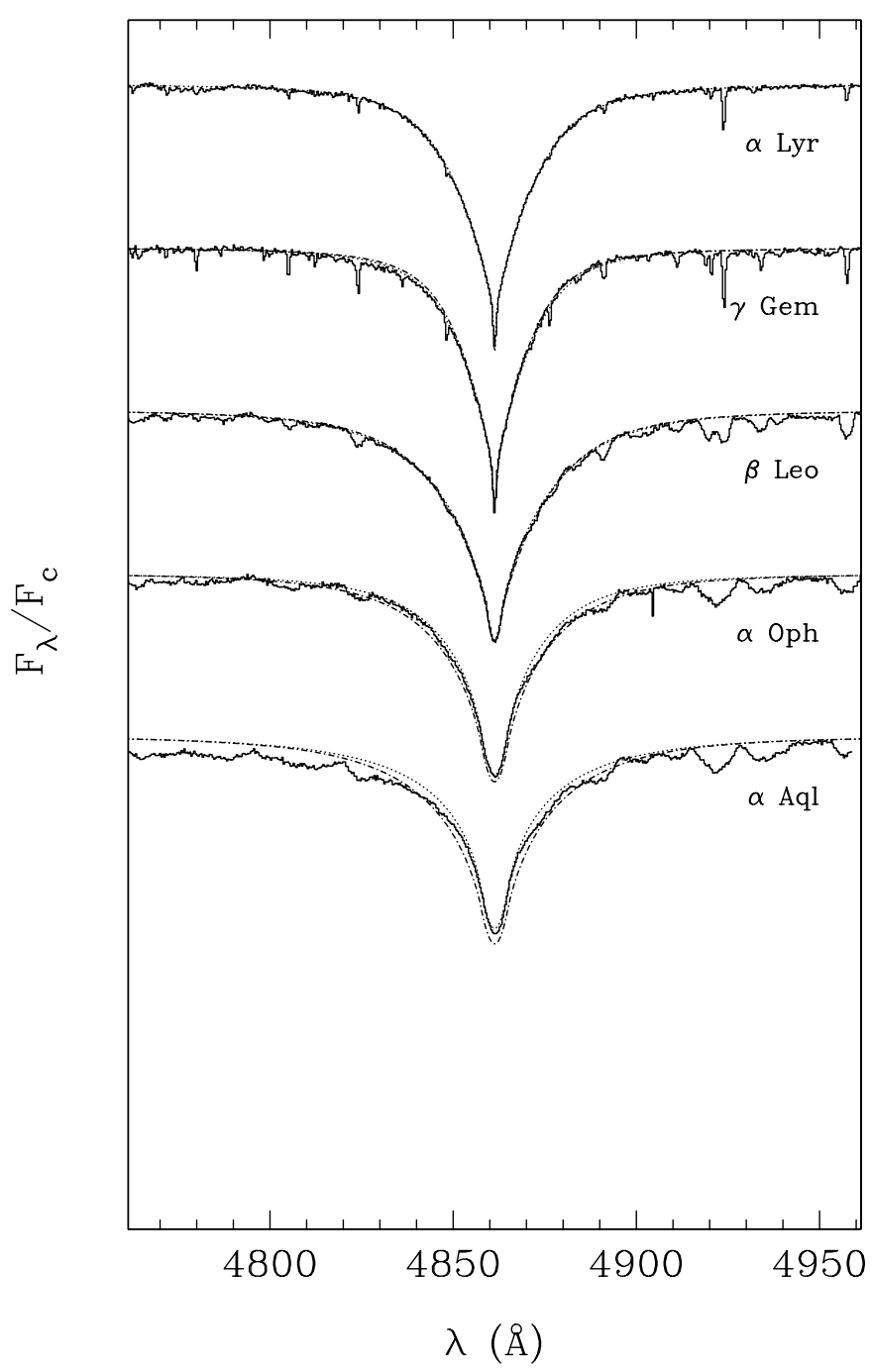

Fig. 4. Same as Fig. 3, but for $\mathrm{H} \beta$ profiles.

and $\log g$ to be considerably extended, from the 4 originally given by Smalley \& Dworetsky (1995), to the 15 presented here. Even when the available optical flux measurements are limited to only $U B V$ magnitudes, the quality of the final $T_{\text {eff }}$ values is good. In some cases, it is the uncertainty of the Hipparcos parallax measurements that limits the accuracy of the $T_{\text {eff }}$ obtained. The stars with IRFM values are mostly in very good agreement with the fundamental values, showing that the two methods are self-consistent and reliable. Since there are more systems with $T_{\text {eff }}$ values from the IRFM (e.g. Blackwell \& Lynas-Gray 1994; Alonso et al. 1995), this method can be used to obtain 'near-fundamental' values, 
provided we avoid binary systems with markedly dissimilar components (Smalley 1993).

Balmer line profiles have been fitted to the fundamental binary systems. To within the errors of the fundamental $T_{\text {eff }}$ values, neither the $\mathrm{H} \alpha$ or $\mathrm{H} \beta$ profiles exhibit any significant discrepancies for the CM and MLT_noOV models. As in previous work, the MLT_OV models are found to be discrepant. Moreover, there are no systematic trends, such as offsets, between results from $\mathrm{H} \alpha$ and $\mathrm{H} \beta$ as long as $\alpha$ in MLT models is chosen small enough (e.g. 0.5). The discrepancies exhibited by the fundamental $T_{\text {eff }}$ stars in GKS99 can be explained by rapid rotation in two cases and by the fact that the Balmer profiles become sensitive to $\log g$ and less sensitive to $T_{\text {eff }}$ in the other two cases. However, for the time being the lack of any stars with fundamental values of both $T_{\text {eff }}$ and $\log g$ in this region precludes the conclusion that there is not a problem with the models in the $T_{\text {eff }}$ range $8000 \sim 9000 \mathrm{~K}$.

Acknowledgements. This work has made use of the sIMBAD data base, operated at the CDS, Strasbourg, France, NASA's Astrophysics Data System (ADS) Abstract and Article Service, and the INES (IUE Newly Extracted Spectra) archive. This work has also made use of the hardware and software provided at Keele by the PPARC Starlink Project. The referee, Mike Dworetsky, is thanked for his helpful comments on the original manuscript. Rebecca Gardiner's PhD studentship was funded by EPSRC. Friedrich Kupka acknowledges support by the project Turbulent convection models for stars, grant P13936-TEC of the Austrian Fonds zur Förderung der wissenschaftlichen Forschung.

This publication makes use of data products from the Two Micron All Sky Survey, which is a joint project of the University of Massachusetts and the Infrared Processing and Analysis Center/California Institute of Technology, funded by NASA and the NSF.

\section{References}

Alonso, A., Arribas, S., \& Martínez-Roger, C. 1995, A\&A, 297, 197 Andersen, J. 1991, A\&AR, 3, 91

Barlow, D. J., Scarfe, C. D., \& Fekel, F. C. 1998, AJ, 115, 2555

Berthet, S. 1990, A\&A, 236, 440

Blackwell, D. E., \& Lynas-Gray, A. E. 1994, A\&A, 282, 899

Blackwell, D. E., \& Shallis, M. J. 1977, MNRAS, 180, 177

Burnashev, V. I. 1985, Abastumanskaya Astrofiz. Obs. Bull., 59, 83 [III/126]

Canuto, V., \& Mazzitelli, I. 1991, ApJ, 370, 295

Canuto, V., \& Mazzitelli, I. 1992, ApJ, 389, 724

Carnochan, D. J. 1979, Bulletin d'Information du Centre de Données Stellaires, 17, 78

Castelli, F., \& Kurucz, R. L. 1994, A\&A, 281, 817

Castelli, F., Gratton, R. G., \& Kurucz, R. L. 1997, A\&A, 318, 841

Ciardi, D. R., van Belle, G. T., Akeson, R. J., et al. 2001, ApJ, 559, 1147

Crawford, D. L. 1975, AJ, 80, 955

Crawford, D. L., \& Barnes, J. V. 1970, AJ, 75, 978

Epchtein, N., Deul, E., Derriere, S., et al. 1999, A\&A, 349, 236 [B/denis]

ESA 1997, The Hipparcos and Tycho Catalogues, ESA SP-1200

Flower, P. J. 1996, ApJ, 469, 355

Gardiner, R. B. 2000, Ph.D. Thesis, University of Keele

Gardiner, R. B., Kupka, F., \& Smalley, B. 1999, A\&A, 347, 876 (GKS99)
Gezari, D. Y., Schmitz, M., \& Mead, J. M. 1987, Catalog of Infrared Observations, NASA Ref. Publ. 1196

Glushneva, I. N., Kharitonov, A. V., Knyazeka, L. N., \& Shenavrin, V. I. 1992, A\&AS, 92, 1

Gray, D. F. 1992, Observation and Analysis of Stellar Photospheres (Cambridge University Press)

Hanbury Brown, R., Davis, J., \& Allen, L. R. 1974, MNRAS, 167, 121

Hayes, D. S., \& Latham, D. W. 1975, ApJ, 197, 593

Heiter, U., Kupka, F., van't Veer-Menneret, C., et al. 2002, A\&A, 392, 619

Howarth, I. D., Murray, J., Mills, D., \& Berry, D. S. 1997, Starlink User Note 50

Jamar, C., Macau-Hercot, D., Monfils, A., et al. 1976, ESA SR-27 [III/39A]

Johnson, H. L., \& Mitchell, R. I. 1975, Rev. Mex. Astron. Astrofís., 1, 299

Jordi, C., Ribas, I., Torra, J., \& Giménez, A. 1997, A\&A, 326, 1044

Kharitonov, A. V., Tereshchenko, V. M., \& Knyazeva, L. N. 1988, Alma-Ata, Nauka, 484 [III/202]

Kupka, F. 1996 in Model Atmospheres and Spectrum Synthesis, ed. S. J. Adelman, F. Kupka, \& W. W. Weiss, ASP Conf. Ser., 108, 73

Kurucz, R. L. 1993, CD-ROM 13, SAO

Kurucz, R. L., \& Bell, B. 1995, CD-ROM 23, SAO

Latham, D. W., Nordström, B., Andersen, J., et al. 1996, A\&A, 314, 864

Macau-Hercot, D., Jamar, C., Monfils, A., et al. 1978, ESA Special Report 28 [II/86]

Mills, D., Webb, J., \& Clayton, M. 1997, Starlink User Note 152.4

Moon, T. T. 1985, Commun. Univ. London Obs., 78

Mountain, C. M., Leggett, S. K., Selby, M. J., Blackwell, D. E., \& Petford, A. D. 1985, A\&A, 151, 399

Nordström, B., \& Johansen, K. T. 1994, A\&A, 291, 777

Peterson, D. M. 1969, SAO Spec. Rept., 293

Petford, A. D., Blackwell, D. E., Booth, A. J., et al. 1988, A\&A, 203, 341

Popper, D. M. 1980, ARA\&A, 18, 115

Popper, D. M. 1984, AJ, 89, 1057

Ribas, I., Giménez, A., Torra, J., Jordi, C., \& Oblak, E. 1998, A\&A, 330,600

Ribas, I., Jordi, C., \& Torra, J. 1999, MNRAS, 309, 199

Skrutskie, M. F., Schneider, S. E., Stiening, R., et al. 1997, in The Impact of Large Scale Near-IR Sky Surveys, ed. F. Garzon, N. Epchtein, A. Omont, B. Burton, \& P. Persi, Astrophysics and Space Science Library Series, vol. 210 (Kluwer, Dordrecht), 25

Smalley, B. 1992, Ph.D. Thesis, University of London

Smalley, B. 1993, MNRAS, 265, 1035

Smalley, B., \& Dworetsky, M. M. 1995, A\&A, 293, 446

Smalley, B., \& Kupka, F. 1997, A\&A, 328, 349 (SK97)

Smalley, B., Smith, K. C., \& Dworetsky, M. M. 2001, UCLSYN Userguide

Smith, K. C. 1992, Ph.D. Thesis, University of London

Thompson, G. I., Nandy, K., Jamar, C., et al. 1978, The Science Research Council, U.K. [II/59B]

Torres, G., Stefanik, R. P., Andersen, J., et al. 1997, ApJ, 114, 2764

van Altena, W. F., Lee, J. T., \& Hoffleit, E. D. 1991, The General Catalogue of Trigonometric Stellar Parallaxes: a Preliminary Version (Yale University Observatory, New Haven)

van Belle, G. T., Ciardi, D. R., Thompson, R. R., Akeson, R. L., \& Lada, E. A. 2001, ApJ, 559, 1155

van't Veer-Menneret, C., \& Megessier, C. 1996, A\&A, 309, 879

Vidal, C. R., Cooper, J., \& Smith, E. W. 1973, ApJS, 25, 37 